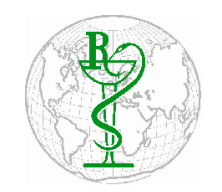

INDO GLOBAL JOURNAL OF

PHARMACEUTICAL SCIENCES

ISSN 2249- 1023

\title{
The Chromosomal \& Molecular Aspect of Mental Retardation: A Review
}

\author{
Kumari R ${ }^{1^{*}}$, Tiwari $\mathrm{M}^{1}$, Agrawal A ${ }^{1}$, Upadhyaya O.P. ${ }^{2}$ \\ ${ }^{1}$ Department of Kriya Sharir, Faculty of Ayurveda, Institute of Medical Sciences, Banaras Hindu University, Varanasi, UP, India \\ ${ }^{2}$ U.S.H.C.C., IMS, Banaras Hindu University, Varanasi, UP, India
}

Address for Correspondance: Rinki K Verma, rinkiv3@gmail.com

\begin{abstract}
Globally, 1-3\% of the population affected by mental retardation (MR), is biggest unsolved clinical problem in society, which creates significant challenges for medicos and scientists. Biological vision of MR is also so complicated due to unexpected heterogeneity of disorders. Analyses of more than 1000 Online Mendelian Inheritance in human (OMIM) database entries and 900 pulse gene has been identified for MR. Genetic factors as well as environmental factors both are responsible for impairment of function of central nervous system and block their function. Cytogenetic system detect the chromosomal aberrations in the identification of MR. However, scientists are able to detect the microscopic abnormalities and several other factors which are responsible for MR. Genetic counselling that can improved MR. Therefore, clinical approaches having aims to develop a new therapeutic application to solve this neurological disorder through direct neurochemicals and biochemical implausible factors which significantly interact at the genetics level, In this review article we have few point out the molecular and genetic vision with chromosomal abnormalities which have a significant role in the dysfunction of CNS in the MR patients. (c) 2014 iGlobal Research and Publishing Foundation. All rights reserved.
\end{abstract}

KEYWORDS: Genetics; Chromosome; CBS; MTHFR; Mental Retardation.

\section{INTRODUCTION}

Epidemiological studies suggests that prevalence of mental retardation (MR) is highly variable, approximately $30-50 \%$ of population, due to different ethnic, geographical and racial aspects [1].Mental retardation is a complex, heterogeneous disorder and dysfunctions of central nervous system. It may be defined as a failure to develop a sufficient cognitive and adaptive level [2]. DSM IV represent the range of disorder with the significant difference on the basis of "Full Scale Intelligence Quotient (FSIQ)" unit, that is represented by an intelligence quotient (IQ) of mild, moderate and severe i.e,: 55 to $69 ; 40$ to 54 and 25 to 39 respectively [3]. It is characterized by subnormal intellectual functioning with impaired cognitive, linguistic, social abilities and adaptive behaviour, that become manifest during developmental years and act as major delinquent for molecular genetics [4].
During embryogenesis large numbers of extrinsic and intrinsic factors are responsible for impairment of CNS and leads to neuropsychiatric disorder as like MR. Numerous studies have demonstrated the genetic, environmental factors and other known or unknown factors including infection, intoxication during pregnancy, complications of delivery, postnatal infection and trauma factor, all are involved in the destruction of brain activity and also interrupt growth among maturation of the brain [5]. Approximately, 5 to $75 \%$ genetic factors act as causative factor in the development of mental retardation. However, MR can be classified, most efficiently into three subclasses, depending on the period of life during which their causative mechanisms are established; (1) the prenatal; (2) the natal and paranatal; and (3) the postnatal. The first subclass of $\mathrm{MR}$, is a part of genetic pool which determines the 
nature and hereditary transmission normal intelligence [6] while natal and paranatal group having entirely different hereditary mechanism in which genetic factors influence the biochemical and pathological pathway of patients and impaired pathway leads to abnormal cerebral structure. Although genetic factors determine the specific enzymatic activity and also are represent the various metabolic processes of proteins, carbohydrates and lipids, resulting following disorders [7].

- Phenyl-pyruvic disease (defect in protein metabolism) due to the absence of the enzyme which determines the metabolism of phenylalanine.

- Galactosemia (defect in carbohydrate metabolism) and affecting the metabolism of galactose.

- Glycogenosis(defect in carbohydrate metabolism)and affecting the metabolism of glycogen or animal starch.

- When intracellular lipid metabolism of the brain cells is abnormal cause the Tay-Sach's group of cerebral lipoidoses.

- Myelin degenerations is known as cerebral scleroses(a lipoidal defect).

- Gargoylism is a multifaceted disease, maybe involving more than one metabolic system and known as metabolic disorder, which involve in the destruct central nervous system with impaired the brain activity [8].

The cellular dysplasia that includes tuberous sclerosis, neurofibromatosis and cerebral angiomatosis is as a type of third class of MR which is recognized by different clinical features and syndromes, collectively known as the congenital ectodermoses. Genetic, epigenetic and environmental factors interact with each other and are responsible for the variable expression of the disease symptoms [9].

\section{CHROMOSOMAL ABNORMALITIES ASSOCIATED WITH MENTAL RETARDATION}

A chromosome anomaly, abnormality, aberration, mutation (missing), extra and irregular portion of chromosomal DNA. Defective cell division (meiosis or mitosis) forms chromosome anomalies. It can be from an atypical number of chromosomes or a structural abnormality in one or more chromosomes and may be detected or confirmed by karyotypes (cytogenetic system). There are many types of chromosomal anomalies and they can be organized into two basic groups, numerical and structural anomalies.

Evolutionary origins of both autosomes and the sex chromosomes are different in nature and implications made by the distinctly X-chromosome to mental functioning. More than 900 genes and approximately
$3.75 \%$ of all genes on the X-chromosome are responsible for the destruction of mental activity and impaired the ability of brain. Nearly all of the genes that have been found to date have been identified through the analysis of balanced chromosomal aberrations or micro deletion's-linked genes, play a disproportionate role in the development of human intelligence and influence both general intelligence with their relatively specific effects on social-cognition and emotional regulation [10]. In 2004, Online-Mendelian Inheritance in Man recorded 1237 entries for 'mental retardation [11]. Mode of inheritance of mental illness and some other common disorders related to CNS having lack of transparency, so that create complexity with Mendelian and nonMendelian subsets. However, some hypothesis proposed that the additive or interactive effects of several genes, each of small effect results in the Autosomal dominant inheritance is almost entirely connected to syndromic MR. The study of non-specific autosomal dominant of MR arises because of mutations in de novo metabolism and are more difficult as patients usually do not reproduce, and thus no large family history can be traced [12-13].

Chromosomal aberrations have reported in many of the linkage 'Hot spots', are susceptibility loci. Its abnormalities include numerical chromosomal mutation; partial chromosome abnormalities and micro deletions are responsible for up to $28 \%$ of mental retardation. However, it is not possible to visualize all deletions by cytogenetic techniques. Some deletions are too diminutive to detect under a microscope, so-called sub microscopic deletions and causing so-called contiguous gene syndrome. Micro deletions have non-random positions in the genome and tend to cluster in specific regions of gene [14].

Chromosome banding studies can rapidly establish the gross morphological areas disrupted by chromosomal aberrations. Micro deletions can be detected through molecular cytogenetic techniques, such as Fluorescent in situ hybridization (FISH), performed when there is actual clinical doubt of a particular micro deletion syndrome, e.g., Williams-Beuren syndrome (deletions in 7q11), 22q11 deletion syndrome, $1 \mathrm{p} 36$ deletion syndrome, or Smith-Magenis syndrome (deletions in 17p11) and to quickly identify the point in the genome that has been disrupted by a chromosomal aberration, and tag candidate genes [15]. Some advance techniques such as "Genomic microarrays" with resolution 1010000 times higher than conventional karyotyping, identifies rare, de novo sub microscopic interstitial imbalances in about $5-20 \%$ of cases of idiopathic MR and multiple congenital abnormalities, depending on the clinical selection of patients. The increased identification of novel micro deletion \& micro duplication syndromes is based on an accurate genotype-phenotype correlation 
Indo Global Journal of Pharmaceutical Sciences, 2014; 4(2): 123-127

i.e., characterized by the association of similar chromosomal aberrations and overlapped clinical presentations between affected patients [16].

Fragile $\mathrm{X}$ syndrome is the most common $\mathrm{X}$ chromosomal disorder with few phenotypic symptoms such as a large head circumference, big ears, a prominent chin, and enlarged testicles. Manifestations of the syndrome may be up and down, so the patients do not reach their full level till puberty. Therefore, it should be included in the differential diagnosis of male with mental retardation, particularly those with a positive family history, but less common in female. Approximately $25 \%$ of all families suffering from familial mental retardation show X-chromosomal inheritance pattern. Recently, it has been identified that genetic defects underlying various types of $\mathrm{X}$-chromosomal retardation are present in both syndromic (Coffin-Lowry syndrome, ATRX syndrome) and non-syndromic MR. A number of reports have described the associations between schizophrenia or bipolar disorder and several fragile sites but yet, there are no any replicates. Furthermore, except at 1q32, linkage studies have not extra support to the suggestion that any of these sites are possible susceptible loci. Therefore, it is possible that these fragile sites either represent at facts or are coincidental findings of no clinical significance [16].

Single gene defect is responsible for many clinically apparent syndrome types of X-linked mental retardation (XLMR). In few patients with bipolar disorder, who had 6 learning disability an unusual translocation $\mathrm{t}(\mathrm{X} ; 12)(\mathrm{q} 24 ; \mathrm{q} 15)$ was reported. There have been several bipolar linkages to markers in the Xq24-27 region; however, these findings have not been consistently replicated. According to European Mental Retardation Consortium, $90 \%$ of all genetic defects causing XLMR by characterization and through the pedigree analysis of families with X-chromosomal MR. Non-syndromic MR, due to defect in more than 20 different genes lead to mental disability. Numerous studies have shown uneven reciprocal translocation $\mathrm{t}(15 ; 18)(\mathrm{q} 13.3 ; \mathrm{q} 22.3)$, trisomy of chromosomes 15 and 18, leads to neuropsychiatric disorder like bipolar disorder and schizoaffective disorder. The schizophrenia linkage has different support to a susceptibility locus in the region 15q13-14. Some slightly proximal region highly associated between Prader-Willi syndrome at 15q11-13 and affective mental disorder with especially arresting the finding of an apparent excess of uniparental disomy cases that is considerable [17]. Another interstitial duplication, $\operatorname{dup}(15)(\mathrm{q} 24 \mathrm{q} 26)$,co-reported as a Lod score i.e. 5.0 with different disease such as panic disorder, social phobia, agoraphobia and joint laxity[18].

\section{EPIGENETIC LINKED TO MENTAL RETARDATION}

Epigenetic of the genome is an important mechanism for the modification of histones proteins with "posttranslational modification" (PTM) in the chromatin. PTMs of histone are variable and co-occur in complex combinations on individual histones and in different nucleosomes. Their combinations are non random, but appear as specific form called a "histone code". PTMs make specific manner of histones by which they form a meticulous code in individual genes, and regulate the activation or silence of genes. The studies has illustrated distinct the histone-modifying enzymes are complex machinery which regulates the activity of these specific codes. A multiple number of histone-modifying enzymes are present in most of the tissues, including the brain that can add or remove PTMs on specific sites like a process "writers" or "erasers" and knows as "readers" which construe the histone code by recognizing dissimilar histone PTMs. This hotchpotch of "read-write" and "read-erase" mechanisms may multiply or delete the epigenetic marks over particular stretches of DNA, and participate in the complex mechanisms of transcriptional control [19].

Mental retardation syndrome X-linked (ATR-X), alpha thalassemia, KDM5C, PHF6, nuclear receptor-binding SET domain containing 1 (NSD1), and CREB binding protein (CBP) have been implicated. These mutations are thought to have a strong impact on the activity of the genes and alter their functions in the brain. ATR-X is a protein coded by the X-linked gene atrx, whose mutations can result in ATR-X syndrome; a disorder characterized by severe mental retardation, microcephaly, seizures and delayed growth. Another Xlinked protein is $\mathrm{KDM} 5 \mathrm{C}$, contains a PHD that recognizes trimethylated $\mathrm{H} 3 \mathrm{~K}$ 9, and a $\mathrm{JmjC}$ domain, which catalyses demethylation of H3K4. Since trimethylation of $\mathrm{H} 3 \mathrm{~K} 9$ represses gene transcription while trimethylation of $\mathrm{H} 3 \mathrm{~K} 4$ activates the gene and their binding of $\mathrm{KDM} 5 \mathrm{C}$ acts synergistically to repress gene transcription. A point mutation in the PHD finger of KDM5C (A388P) reduces the protein's binding to H3K9 and decreases demethylase activity [20].

NSD1 is another protein involved in epigenetic regulation that acts as either a co-repressor or a coactivator. NSD1 contains a su(var), enhancer-of-zeste, trithorax (SET) domain with histone methyltransferase activity, and multiple PHD domains. Mutations in the PHD fingers are associated with two overgrowth syndromes, Sotos syndrome and more rarely, Weaver syndrome. These both diseases are characterized by, pre and postnatal somatic overgrowth, craniofacial abnormalities, sophisticated bone age, and mild mental 
Indo Global Journal of Pharmaceutical Sciences, 2014; 4(2): 123-127

retardation [21]. CBP is a transcriptional regulator linked to mental retardation in Rubenstein-Taybi syndrome. It has HAT activity and its haplo insufficiency alters brain functions.

Methylation is essential for the epigenetic regulation of this gene expression. $\mathrm{MeCP} 2$ is a methyl-CpG binding domain (MBD) protein that recognizes methylated DNA by selectively binding to methylated $\mathrm{CpGs}$ adjacent to $\mathrm{A} / \mathrm{T}$ sequences. MeCP2 is expressed ubiquitously across human tissues, but is present in high amount in the brain, particularly in neurons. Its level increases during postnatal neuronal development, suggesting a conserved function in the maturation of existing neurons, rather than in the generation of new neuron in human. In neurons, MeCP2 expression peaks after mitosis, and continues to be high in postmitotic neurons in the adult, suggesting that it is also necessary for neuronal function in mature neurons. Approximately $1: 10,000$ in a live female child suffer by the mutations in MeCP2 which linked to Rett syndrome. Rett syndrome is identified by progressive cognitive decline despite normal early infancy and by the development of hand stereotypes and seizures that appear between 6 and 18 months of age [22]

One gene variant in particular, the C677T genotype, of the 5,10 methylenetetrahydrofolate reductase (MTHFR) gene, has been associated with neuropsychiatric disorder and other complex congenital anomalies leads to mental retardation. MTHFR variants codes thermolabile enzyme has been linked to the metabolism of folate. Folate is an essential component for the development of central nervous system during organogenesis and any error leads to abnormal differentiation causes mental retardation. Folic acid deficiency leads to increase plasma homocysteine C677T in an individual's known to increase genetic risk factor and chromosomal instability for various clinical lesions. The various studies have declared the MTHFR and intelligence quotient in down syndromes is associated with "risk factor" in infants in homozygous C677T genotype for severe mental retardation. Although, the role of MTHFR C677T gene polymorphism is not yet properly understood, but it is believed that either paternal or maternal factor contribute the etiologies of congenital "birth defects" leads to the mental retardation. MTHFR catalyses irreversible conversion of 5,10-methylenetetrahydrofolate to 5methyltetrahydrofolate, which is the methyl donor for methionine synthesis from homocysteine and participates in S-adenosyl methionine synthesis and DNA methylation. MTHFR genes and homocysteine concentration also affect the different mental performance and school performance of child [23]

Another gene, cystathionine $\beta$-synthase (CBS) catalyses the condensation of serine and homocysteine to form cystathionine. The studies have been shown the abnormality in CBS activity is manifested in two major clinical conditions, viz. hyperhomocysteinemia and homocystinuria. Insufficiency in CBS activity may lead to hyperhomocysteinemia, which is considered to be an independent risk factor for arteriosclerosis. In addition to that, since homocysteine is vasculotoxic as well as neurotoxic, hyperhomocysteinemia predisposes to cardiovascular disorder (CVD) and cognitive dysfunctions. Crass deficiency in CBS activity is associated with homocystinuria, an inborn recessive metabolic disorder. The major pathologic abnormalities associated with homocystinuria include thromboembolism, ectopia lentis, osteoporosis, mental retardation (MR) and other neurological \& psychiatric abnormalities. The neurological malfunctioning can be ascribed to the oxidation of excess homocysteine to homocysteic acid, which interacts with the N-methyl-Daspartate receptor, causing an excessive calcium influx and free radical production, thereby leading to neurotoxicity [24].

\section{CONCLUSON}

MR is CNS dysfunction by the lack of homogeneous form of various neurotransmitters, but still there is a no any strong evidence to prove their existing pathogenic mechanisms at the neuronal and molecular level. MR is one of the CNS disorder due to impairment of genes function, regarding translational and transcriptional because the candidate gene expression occur in the protein form which interact with different neural molecules of brain for causing neuropsychiatric illness through discontinuous transmission of neural circuitries which affected circadian rhyme or clock of both human and animal. So, there are number of chromosomal abnormalities and defect the molecular mechanism. The hypothesis for the management large number of pharmacological approaches determine to form number of anti psychical drugs, ranging from the Monoamine Oxidase Inhibitors to Tricyclics etc, Due to adverse side effect of modern anti psychical drugs, should develop and utilize the "Indian Medicine" such as Ayurveda treatment as medhya rasayan, other herbal based product and panchkarma therapy.

\section{REFERENCES}

1. Argentaro, A., Yang, J. C., Chapman, L., Kowalczyk, M. S., Gibbons, R. J., Higgs, D. R.,et al. Structural consequences of disease-causing mutations in theATRX-DNMT3-DNMT3L (ADD) domain of the chromatin-associated protein ATRX. Proceedings of the National Academy of Sciences of the United States of America 2007; 104:11939-11944.

2. Bi, W., Park, S.-S., Shaw, C. J., Withers, M. A., Patel, P. I., Lupski, J. R. Reciprocal crossovers and a positional preference 
Indo Global Journal of Pharmaceutical Sciences, 2014; 4(2): 123-127

for strand exchange in recombination events resulting in deletion or duplication of chromosome 17p11.2. Am. J. Hum. Genet.2003; 73:1302-1315.

3. Carter, J. C., Lanham, D. C., Pham, D., Bibat, G., Naidu, S., \& Kaufmann, W. E. Selective cerebral volume reduction in Rett syndrome: A multiple-approach MR imaging study. American Journal of Neuroradiology.2008;29:436-441.

4. Curry, C.J., Stevenson, R.E., Aughton, D.,Byrne, J., Carey, J.C., Cassidy, S., Cunniff,C., Graham, Jr. J.M., Jones, M.C., Kaback,M.M., Moeschler, J., Schaefer, G.B.,Schwartz, S., Tarleton, J. and Opitz, J. Evaluation of Mental Retardation. Recommendations of a Consensus Conference. American Journal of MedicalGenetics.1997;72:468-477.

5. Ephrem Engidawork and Gert Lubec.Molecular changes in fetal Down syndrome brain Journal of Neurochemistry.2003;84:895-904.

6. Ferguson-Smith MA, Handmaker SD. Observationon the satellite human chromosomes. Lancet.1961;1:638-640

7. Flint J, Wilkie AO, Buckle VJ, Winter RM, Holland AJ,McDermid HE. The detection of subtelomericchromosomal rearrangements in idiopathic mentalretardation. Nat Gene.1995;9:132-140.

8. Frosst, P., Blim, H. J., Milos, R., Goyette, P., Sheppard, C. A., Matthews, R. G., Boers, G.J.H., den Heijer, M., Kluijtmans, L.A.J., van den Heuvel, L. P. \& Rozen, R.A candidate genetic risk factor for vascular disease: A common mutation in methylenetetrahydrofolate reductase. Nat. Genet. 1995;10:111113.

9. Graves J.A., Gecz, J. and Hameister, H. 2002 Evolution of the human $\mathrm{X}-\mathrm{a}$ smart and sexy chromosome that controls speciation and development. Cytogenet. Genome Res. 99 141145.

10. Gijsbers AC, Lew JY, Bosch CA, Schuurs-Hoeijmakers JH, van Haeringen A, den Hollander NS, Kant SG, Bijlsma EK, Breuning $\mathrm{MH}$, Bakker E, Ruivenkamp CA.A new diagnostic workflow for patients with mental retardation and/or multiple congenital abnormalities: test arrays first. Eur J Hum Genet. 2009; 17:1394-1402.

11. Gibbons, R. J., S. Bachoo, D. J. Picketts, S. Aftimos, B. Asenbauer, J. Bergoffen, S. A. Berry, N. Dahl, A. Fryer, K. Keppler, K. Kurosawa, M. L. Levin, M. Masuno, G. Neri, M. E. Pierpont, S. F. Slaney, and D. R. Higgs.Mutations in transcriptional regulator ATRX establish the functional significance of a PHD-like domain. Nat. Genet. 1997;17:146148.

12. Guéant-Rodriguez RM, Rendeli C, Namour B, Venuti L, Romano A, Anello G, Bosco P, Debard R, Gérard P, Viola M, et al.Transcobalamin and methionine synthase reductase mutated polymorphisms aggravate the risk of neural tube defects in humans. Neurosci Lett.2003;344:189-192.
13. Herbst DS, Miller JR. Nonspecific X-linked mental retardation II: the frequency in British Columbia.Am J Med Genet.1998;7:461-9.

14. Hagerman RJ, Hagerman PJ.Fragile X syndrome: a model ofgene-brain-behavior relationships. Mol Genet Metab.2001;74:89-97.

15. Hobbs CA, Sherman SL, Yi P, Hopkins SE, Torfs CP, Hine RJ, Pogribna M, Rozen R, James SJ.Polymorphisms in genes involved in folate metabolism as maternal risk factors for Down syndrome. Am J Hum Genet. 2000;67:623-630.

16. Kahler SG, Fahey MC 2003Metabolic disorders and mental retardation. Am J Med Genet C Semin Med Genet.2003;117:31-41.

17. Irwin, S. A., Swain, R. A., Christmon, C. A., Chakravarti, A., Weiler, I. J. andGreenough, W. T. Evidence for altered Fragile$\mathrm{X}$ mental retardation protein expression in response to behavioral stimulation. Neurobiol. Learn. Mem.2000;73: 8793.

18. Jamie M. Kramera, b, Hans van Bokhovena. Genetic and epigenetic defects in mental retardation. The International Journal of Biochemistry \& Cell Biology. 2009;41:96-107.

19. Koekkoek SK, Yamaguchi K, Milojkovic BA, Dortland BR, Ruigrok TJ, Maex R, De Graaf W, Smit AE, VanderWerf F, Bakker CE, Willemsen R, Ikeda T, Kakizawa S, Onodera K, Nelson DL, Mientjes E, Joosten M, De Schutter E, Oostra BA, Ito $\mathrm{M}$, et al. Deletion of FMR1 in Purkinje cells enhances parallel fiber LTD, enlarges spines, and attenuates cerebellar. Neuron. 2005;3:339-52.

20. Mandel, J.-L., Hagerman, R., Froster, U., Brown, W. T., Jenkins, E. C., Jacobs, P., Turner, G., Lubs, H., Neri, G.Fifth International Workshop on the Fragile X and X-Linked Mental Retardation. Am. J. Med. Genet. 1992;43:5-27.

21. Lehrke, R.,Theory of X-linkage of major intellectual traits. Am. J. Ment. Defic. 1972;76:611-619.

22. Luckasson, R., Borthwick-Duffy, S., Buntinx,W. H. E., Coulter, D. L., Craig, E. M., Reeve, A., Schalock, R. L., Snell, M. E., Spitalnick, D. M.,Spreat, S., \& Tasse, M. J.Mental retardation: Definition, classification, and systems of supports(10thEd.). Washington DC: American Associationon Mental Retardation.2002.

23. Orendac M, Muskova B, Richterova F, Zvarova J, Stefek M, Zaykova E, Stribrny J, Hyanek J, Kraus JP, Kozich V.Mutation C677T in MTHFR gene and polymorphism 844ins68 in the CBS gene: Risk factor for peripheral arterial occlusive disease? Neth J Med.1998; 47.

24. Pascual, J., M. Martinez-Yamout, H. J. Dyson, and P. E. Wright.Structure of the PHD zinc finger from human Williams-Beuren syndrome transcription factor. J. Mol. Biol.2000;304:723-729.

Indo Global Journal of Pharmaceutical Sciences( ISSN 22491023 ; CODEN- IGJPAI; NLM ID: 101610675) indexed and abstracted in EMBASE(Elsevier), SCIRUS(Elsevier),CABI, CAB Abstracts, Chemical Abstract Services(CAS), American Chemical Society(ACS), Index Copernicus, EBSCO, DOAJ, Google Scholar and many more. For further details, visit 